\title{
Current Advances in the Use of Therapeutic Hypothermia
}

\author{
Moderator: Neeraj Badjatia, $\mathrm{MD}^{1}$ \\ Participants: Christopher J. White, MD, ${ }^{2}$ Abbot Laptook, MD, ${ }^{3}$ \\ and Markus Föedisch, $\mathrm{MD}^{4}$
}

Temperature management and therapeutic hypothermia is being investigated in stroke, subarachnoid hemorrhage, myocardial infarction, and neonatal encephalopathy. Previous clinical studies have provided support for the use of hypothermia in these clinical conditions, and new studies are currently underway to establish safety and efficacy of this therapeutic intervention. Thus, this particular session brought together experts in the field of therapeutic hypothermia to discuss its use in several important patient populations. Dr. Neeraj Badjatia from the University of Maryland School of Medicine discussed antishivering strategies targeting stroke and subarachnoid hemorrhage. This lecture emphasized thermoregulatory systems and systematic approaches toward shiver control during cooling in patients with acute stroke. Various pharmacological strategies are currently used for shiver control, including drug combinations and counterwarming approaches. Dr. Christopher J. White of the Ochsner Medical Center emphasized the use of hypothermia in myocardial infarction. Myocardial infarction is an extremely important clinical problem, and the use of hypothermia to reduce ischemic injury and reperfusion-injury is currently being assessed in both preclinical and clinical studies. Previous clinical studies including a prospective randomized trial reported that postreperfusion cooling provided no significant improvement. However, recent investigations are concentrating on hypothermia treatment initiated before reperfusion, and these have been very promising. Dr. Abbot Laptook, Brown University, updated the attendees on therapeutic cooling and neonatal hypoxic ischemic encephalopathy. Results from published multicenter trials have emphasized the benefits of therapeutic hypothermia in newborn hypoxic ischemic encephalopathy patients, and issues regarding implementation of hypothermia on transport to hospitals are being evaluated along with combination approaches. Dr. Markus Föedisch from Bonn, Germany, discussed the use of brain damage markers to determine how best to utilize hypothermia after cardiac arrest. Dr. Föedisch updated the attendees regarding the cool brain register as well as the effects of cooling on available biomarkers, including neuron-specific enolase and S100B. This session had an extensive question-and-answer exchange and provided new information re- garding the advances being made in the use of hypothermia in these clinical conditions.

Question: You mentioned, and I would, agree that there is no actual threshold that we should pay attention to in terms of prognostication. Overall prognostication biomarkers are just one piece of the data. But what you seem to be indicating is that perhaps we should be looking at the trend lines. You have individual lines there, but have you looked at overall trend lines and looked at deltas in terms of where you start and where you end up at 72 hours to help determine prognosis?

Dr. Markus Föedisch: As I told you, it is very preliminary. We have only had 25 patients up to now. What we have seen is that a singular measurement of neuron-specific enolase (NSE) values cannot help you at all. What we have seen is that you have constant increasing values when we have a bad prognosis, even if you treat a patient with hypothermia. But you see normally in the patients where a good prognosis is expected to decrease values is not earlier than $48-72$ hours. We don't use those elevated NSE values as a marker or decision maker for stopping the therapeutic approach. It is only one piece.

Question: I would like to follow up on that. I assume that with these changes in NSE, you are assuming that you are seeing selective neuronal necrosis. I assume that is the premise, correct? The biomarker neuron-specific enolase is coming neuronal. So you would assume it's lost in the systemic blood as a reflecting neuronal necrosis, cell death.

Dr. Markus Föedisch: Yes.

Question: Do you ever have any patients die, and do you do postmortems to confirm the correlation between the neuronspecific enolase and the burden of cell death? That would be fascinating.

\footnotetext{
${ }^{1}$ Department of Neurology, University of Maryland, Baltimore, Maryland.

${ }^{2}$ Department of Medicine, Ochsner Medical Institutions, New Orleans, Louisiana.

${ }^{3}$ Department of Pediatrics, Brown University, Providence, Rhode Island.

${ }^{4}$ Department of Anaesthesiology, Evangelische Kliniken Bonn, Bonn, Germany.
} 
Dr. Markus Föedisch: Not in $100 \%$. We have patients with high NSE levels who had survived, even in the time before we cooled them for 72 hours. We also have patients with low NSE values who didn't survive and died because of brain death.

Comment: I saw that in your data, and that brings us back then to the question, what is the most robust marker? Other people in neuron-specific discovery are looking at ubiquitization. You see how each one is a very robust tool to look at neuronal cell death. Interestingly, glial fibrillary protein degradation products are also rising to the top. I think what you are doing is extremely important, but I think I would expand the window or perhaps use other biomarkers. You may also consider in those patients that die, perhaps a postmortem. Because I believe that the Germans are much more effective in gaining postmortem analysis than we are in America.

Dr. Markus Föedisch: But I have to confirm that the only reliable biomarkers we can obtain in our hospital, also in the university hospital studies, are NSE and S100B. We get the NSE values within 4 hours and the S100B within 30 minutes. We don't have access to other biomarkers in the therapeutic approach of our patients.

Dr. Neeraj Badjatia: The other way to look at this in lieu of those restrictions is determining correlations between magnetic resonance imaging (MRI) biomarkers with serum biomarkers. My own experience with NSE and MRI is that the correlation isn't great, which brings out what you are bringing up, and that is what we are actually measuring with the NSE. But I think other markers like GFAP or breakdown products might be interesting to look at. I tend to use MRI probably more reliably than serum biomarkers, along with everything else that we think about in terms of prognosis.

Dr. Markus Föedisch: We still have a problem with the MRI because the MRI has no place for ventilation there still. So we cannot make an MRI image during the therapy of ventilated patients. Our MRI is outside of the hospital, and so we really have problems doing that.

Question: Compliments to all the speakers. I have a question for Dr. Laptook. You explained that a lot of ongoing trials for hypothermia duration is tested and combined with xenon. I think those are important developments. You also said that another trial is ongoing comparing hypothermia to normothermia. I was wondering if you would comment on whether it is even still ethical to do that. I know it is always difficult to pose this kind of question, but it seems sometimes that we are just going to continue doing trials until we finally come up with a negative one. How much evidence do we really need?

Dr. Abbot Laptook: I don't think we have any evidence for using hypothermia after 6 hours of age. The data in animal work actually suggest that beyond 8 hours of age is not effective. So, in translating that into the clinical arena, I think we are well grounded in doing a randomized trial comparing hypothermia to an actively controlled normothermic arm where the temperature is monitored continuously watching for hyperthermia with algorithms in place if the temperature goes up. But I don't think we have any data to tell us to use it beyond 6 hours of age. All the trials that have been done have been done with infants, and they are all at less than 6 hours of age. And that is what the animal data basically supported.

Question: I guess my question would be whether we would need still more trials before 6 hours of age, as opposed to after, because it still is not standard of care and recommended by the various societies to use hypothermia in the study group and in these patients meeting the criteria of the studies that were published so far.

Dr. Abbot Laptook: I think that hypothermia is being widely disseminated in the neonatal community. There are surveys being published that are showing that it is being adopted by 90-95\% of tertiary centers. So there are very few places that are not providing it, or if they are not providing it, they are transferring their patients to centers that do provide it. So in terms of a normothermic arm at less than 6 hours, yes, I would think that has some ethical dilemmas, and in our current trial optimizing hypothermia regimens, the reference group is a temperature of $33.5^{\circ}$ for 72 hours.

Comment: If you have an adaptation rate of $95 \%$, then you are doing a much better job than us adult doctors. So compliments.

Question: I have a question for the cardiologist. The cardiologists pull out the catheter after 1 hour if they have done their job. That's quite remarkable if they ordered it after cardiac arrest in neurological for intervention. We do it much longer. So why do you pull it out so early; is that only logistic? Or is there an idea behind, based on physiological activity?

Dr. Neeraj Badjatia: Regarding the duration of the catheter in most cardiac subjects, it seems like the cooling catheter is in for a short duration, about an hour, versus other populations, where it is much longer.

Dr. Christopher J. White: So the short duration of the catheter is empiric. I have no knowledge of the study design from the Goteborg group. I believe that they had a philosophy that said they wanted to manage their patients in a very narrow zone. So it was an arbitrary decision because they could not find evidence of any benefits to longer therapy. They simply said: this suits our practice; this suits the construct of the trial. So I think it does raise a reasonable hypothesis to test. If they show benefits in the larger trial that is being run now, which is likely they will, then that will leave open the question of whether it would be better to have the longer, 24-hour cooling period. Perhaps in bigger, larger myocardial infarctions there will be some advantage. But I think the first thing that will happen will be to put the stake in the ground for 1 hour of cooling.

Comment: I think I asked David Erlinge this question last year, that with brain injury a longer degree of cooling might be important. In other words, is there a big difference between the central nervous system and heart tissue? Basically he felt that the inflammatory response was quite different between the two tissues. You do not see such a robust secondary injury mechanism that we see in the brain versus the heart. I thought that was a very interesting comment and one that we have to consider and investigate in the future. 
Comment: I also think that the pig studies showed no additional benefit after an hour. It was data from the animal studies that made them reach the conclusion to do the hour.

Dr. Christopher J. White: Just so. I think you're right, but just remember that there are no animal models of a 40-year process of adult atherosclerosis in the heart. We develop coronary lesions with collaterals and diffuse coronary disease and other problems. Often times, some of the animal experiments are clean, and they're nice, but they may not reflect what happens in human populations.

Question: With the NIH and the FDA now increasingly accepting Bayesian-adapted clinical trial designs, why are we not seeing more Bayesian-adapted designs in the clinical trials that are being planned? Or are we?

Dr. Christopher J. White: So I've been to the FDA twice in the last couple of years with hypothermia trials, and they have been very difficult. I've spent 3 months trying to answer questions regarding protocols. So the FDA has not created a facilatory environment to investigate the clinical application of mild hypothermia at all. It's really hard, actually. Then, I go to my IRB, who is unfamiliar with this field, and they won't allow any protocol that prolongs the door-to-balloon time over 90 minutes. I'm telling them, as an expert, that I think it's reasonable to do this. I recommend testing hypothermia even with prolongation of the door-to-balloon time, and they won't allow it. So the regulatory environment to do this kind of clinical investigation is really tough.

Dr. Abbot Laptook: One of the things that we recognized when we started the late hypothermia trial is that we needed to provide the neonatal community with some evidence of whether you should or should not use this therapy beyond 6 hours. We also anticipated that as therapeutic hypothermia was disseminated, it would be harder and harder to get babies into this trial. So that study was designed and preplanned with Bayesian analysis. The sample size is only 168 . It does not have the sample size that the prior trials had. But then again, it's within a network, within the NIH, so it's not exactly the same thing.

Question: I missed that. Is there an FDA person here that you were addressing?

Comment: We have an FDA/NIH cosponsored grant looking at adaptive clinical trials in phase 3 confirmatory studies and the number of designs that we've worked on with the FDA at the table. It turns out that the agency does not think and breathe in one fell swoop and have all the same attitudes. There is a lot of conciliatory and very constructive work. Some of the best clinical trials come out of partnerships with the FDA.

Dr. Christopher J. White: I think our federal regulatory environment is the reason that my field waits for things to happen in Europe and Canada and other parts of the world before we can bring cutting-edge technology to my patients. Last week I recommended a patient go to Canada for a therapy. Because our agencies are so risk-averse, we stunt new ideas. We do not aggressively go after the frontiers in science. We are happy to let others take the risks and, of course, reap the benefits for their patients before we allow the technology to enter the United States.

Comment: What I'm really saying is that it is not uniform across the agency. I'm not saying that doesn't happen, but the question was, why aren't we seeing more adaptive Bayesian work being done? I don't think it is the FDA that's stopping it. FDA has a guideline for these trials, and they are trying to work out the best way to make those methodologies work in well-controlled confirmatory studies. So, there is an opportunity at least within the agency. We've taken a duration of hypothermia in a cardiac arrest study to them that is Bayesianadaptive in design, and they've been very supportive. We have another application that is also Bayesian-adaptive, and for hypothermia and spinal cord injury, of which the FDA has been very supportive. So there are opportunities, and I think this is a way to make clinical trials better in this area, and there are a lot of potential barriers to it. But the perception of a monolithic barrier is probably a barrier in and of itself.

Question: This question is regarding ST segment elevation myocardial infarction (STEMI) patients. Do you believe that patients who present within an hour are different than patients who present within, let's say, 12 hours? Or if the patient presents within 1 hour, you could think about freezing down the process and simply stopping it to give the interventionist a chance to do his procedure and therefore limit the amount of damage, as opposed to the patient who presents at 12 hours, when the horse is a little bit out of the barn. Now you are thinking about whether or not you can, in effect, remodel that way. The question is whether or not your duration of hypothermia would differ in the two groups, and would you change the temperature setting? If you really want to freeze it, should you go lower?

Dr. Christopher J. White: So there are three answers to this question. I think now we can articulate two injury models. One is the ischemic injury time and one is reperfusion injury. So even at 12 hours, if we cool one and not cool the other, we will potentially have a facilitative reperfusion or less injury with reperfusion at 12 hours if viable myocardium remains. The ischemic time issue will be a 1-hour patient issue. The problem with the 12-hour patient is that the vessel repeatedly occludes and then recanalizes, which causes much variation in injury from one patient to the next. That's why the human studies are so difficult to perform. In terms of the depth of hypothermia, our experience has been with $32^{\circ}$ to $34^{\circ} \mathrm{C}$. If we go lower than $32^{\circ} \mathrm{C}$, we have had some arrhythmia problems. We value the hypothermia systems that self-regulate temperature so that we do not overshoot with cooling or let patients become lower than we want.

Comment: Just wanted to mention that when we think about human disease, we think about prevention, protection, and repair. A new exciting area for temperature management and therapeutic hypothermia is the ability of temperaturedependent regenerative processes. Our own lab and others are now looking at the importance of mild hypothermia and mild hyperthermia on neuronal neurogenesis. We now know that the immature cells are produced in different neurogenic areas of the brain, such as the subgranular zone and the dentate gyrus. It takes about 14 days for these immature cells 
to become adult cells and integrate into the hippocampal circuitry and become involved in learning and memory. These immature cells are very sensitive to excitotoxicity, free radicals, and all the things that we know affect adult neuron survival. So it's kind of interesting to think about durations of hypothermia in terms of not only protecting against adult neuronal cell death, but also the ability to enhance reparative strategies such as neurogenesis. This effect of hypothermia may be important in our infant pediatric population where we think there may actually be more neurogenesis. Again, this is something to consider regarding temperature-mediated events. It involves both protection and repair.

Dr. Christopher J. White: I would just say that we have had trial after trial in acute myocardial infarction where we actually give as close to stem cells as we can. There is a tiny signal of benefit in the heart, but very little. So your hypothesis that perhaps we are putting these cells in a harmful or unattractive milieu, this reperfusion milieu may not be the best place to put stem cells, and that perhaps it could be modulated by temperature, I think, is really interesting.

Dr. Abbot Laptook: I can tell you that as one of the centers participating in the optimizing cooling trial in prolonging hypothermia to 5 days, it becomes very difficult to deal with how long we are going to continue this therapy before we are able to get some kind of signal as to what the baby is like before we are able to image the baby. Most neonatal units don't feed babies when their temperatures are down at 33 or 32 degrees, so we are dealing with basic nutritional issues and maintaining them. So conceptually, I can see how it is attractive. Translating it into something that is safe and balances the needs of families and other organ systems might take a bit.

Comment: Our strategy may be to continue restricted periods of hypothermia to at least protect those very immature cells within the first couple of days after injury and then provide proneurogenic molecules that actually enhance neurogenesis.

Dr. Abbot Laptook: I think the advocates of erythropoietin like the concept of using hypothermia early to stop the deleterious processes, get an agent onboard that could promote neurogenesis, angiogenesis and there certainly is encouraging data from animals. I think it is pretty well studied in animal work in terms of the neuroprotective effects in the newborn.

Question: Dr. Föedisch, do you take into account your NSE level in terminating your therapy? When you think the patient is not doing well by some guidelines, say certain levels of NSE, do you stop treatment? Do you do that in Germany?

Dr. Föedisch: We have never done it, and we won't do it. We won't use NSE levels to stop therapy.

\section{Question: So why is it in the guidelines, then?}

Dr. Föedisch: Is it in the American Heart Association guidelines? In my opinion, it's not included in the NSE guidelines. I don't know. I would never, ever stop them. There is no literature, no study out there that can definitely say we can use not only NSE but biomarkers as a definition for withdrawal of the therapeutic approach.

\section{Question: Also, not to use it as supportive evidence?}

Dr. Föedisch: I think, to say it honestly, it is, if ever, only a piece of the whole bunch. If you have multiorgan failure, you have NSE values, and you have a severe low cardiac output syndrome, even after rewarming, then it's only one point. It is not the point at which to decide to stop the therapeutic approach. In our procedures, we take the targeted temperature management up to 7 days. After that, we stop in case of good pulmonary function and adequate sedation, we conduct imaging, and we make the full therapeutic window for another 72 hours. And then possibly, the patient makes the decision or we make the decision. But not earlier.

\section{References}

Alkadri ME, Peters MN, Katz MJ, White CJ. State-of-the-art paper: therapeutic hypothermia in out of hospital cardiac arrest survivors. Catheter Cardiovasc Interv. 2013 [Epub ahead of print]; doi: 10.1002/ccd.24914.

Badjatia N. Fever control in the NICU: is there still a simpler and cheaper solution? Neurocrit Care 2011;15:373-374.

Badjatia N, Carpenter A, Fernandez L, Schmidt JM, Mayer SA, Claassen J, Lee K, Connolly ES, Seres D, Elkind MS. Relationship between $\mathrm{C}$-reactive protein, systemic oxygen consumption, and delayed cerebral ischemia after aneurysmal subarachnoid hemorrhage. Stroke 2011;42:2436-2442.

Choi HA, Ko SB, Presciutti M, Fernandez L, Carpenter AM, Lesch C, Gilmore E, Malhotra R, Mayer SA, Lee K, Claassen J, Schmidt JM, Badjatia N. Prevention of shivering during therapeutic temperature modulation: the Columbia antishivering protocol. Neurocrit Care 2011;14:389-394.

Choi HA, Badjatia N, Mayer SA. Hypothermia for acute brain injury-mechanisms and practical aspects. Nat Rev Neurol 2012;8:214-222.

Helbok R, Kurtz P, Schmidt MJ, Stuart MR, Fernandez L, Connolly SE, Lee K, Schmutzhard E, Mayer SA, Claassen J, Badjatia N. Effects of the neurological wake-up test on clinical examination, intracranial pressure, brain metabolism and brain tissue oxygenation in severely brain-injured patients. Crit Care 2012;16:R226.

Helbok R, Kurtz P, Vibbert M, Schmidt MJ, Fernandez L, Lantigua H, Ostapkovich ND, Connolly SE, Lee K, Claassen J, Mayer SA, Badjatia N. Early neurological deterioration after subarachnoid haemorrhage: risk factors and impact on outcome. J Neurol Neurosurg Psychiatry 2013;84: 266-270.

Kudagi VS, White CJ. Endovascular stents: a review of their use in peripheral arterial disease. Am J Cardiovasc Drugs 2013; 13:199-212.

Laptook AR. Initiating therapeutic hypothermia during transport for encephalopathy: current state and future direction. J Perinatol 2013;33:169-170.

Laptook AR, McDonald SA, Shankaran S, Stephens BE, Vohr BR, Guillet R, Higgins RD, Das A; Extended Hypothermia Followup Subcommittee of the National Institute of Child Health and Human Development Neonatal Research Network. Elevated temperature and 6- to 7-year outcome of neonatal encephalopathy. Ann Neurol 2013;73:520-528.

Natarajan G, Pappas A, Shankaran S, Laptook AR, Walsh M, McDonald SA, Ehrenkranz RA, Tyson JE, Goldberg RN, Bara R, Higgins RD, Das A, Munoz B. Effect of inborn vs. outborn delivery on neurodevelopmental outcomes in infants with 
hypoxic-ischemic encephalopathy: secondary analyses of the NICHD whole-body cooling trial. Pediatr Res 2012;72:414-419. Shankaran S, Barnes PD, Hintz SR, Laptook AR, Zaterka-Baxter KM, McDonald SA, Ehrenkranz RA, Walsh MC, Tyson JE, Donovan EF, Goldberg RN, Bara R, Das A, Finer NN, Sanchez PJ, Poindexter BB, Van Meurs KP, Carlo WA, Stoll BJ, Duara S, Guillet R, Higgins RD; Eunice Kennedy Shriver National Institute of Child Health and Human Development Neonatal Research Network. Brain injury following trial of hypothermia for neonatal hypoxic-ischaemic encephalopathy. Arch Dis Child Fetal Neonatal Ed 2012;97:F398-F404.

Shankaran S, Pappas A, McDonald SA, Vohr BR, Hintz SR, Yolton K, Gustafson KE, Leach TM, Green C, Bara R, Petrie Huitema CM, Ehrenkranz RA, Tyson JE, Das A, Hammond J, Peralta-Carcelen M, Evans PW, Heyne RJ, Wilson-Costello DE, Vaucher YE, Bauer CR, Dusick AM, Adams-Chapman I,
Goldstein RF, Guillet R, Papile LA, Higgins RD; Eunice Kennedy Shriver NICHD Neonatal Research Network. Childhood outcomes after hypothermia for neonatal encephalopathy. N Engl J Med 2012;366:2085-2092.

Weisz G, Smilowitz NR, Parise H, Devaud J, Moussa I, Ramee S, Reisman M, White CJ, Gray WA. Objective simulator-based evaluation of carotid artery stenting proficiency (from Assessment of Operator Performance by the Carotid Stenting Simulator Study [ASSESS]). Am J Cardiol 2013;112:299-306.

White CJ. Acute stroke treatment: carotid "stenters" to the rescue. J Am Coll Cardiol 2011;58:2370-2371.

White CJ, Ramee SR, Collins TJ, Jenkins JS, Reilly JP, Patel RA. Carotid artery stenting: patient, lesion, and procedural characteristics that increase procedural complications. Catheter Cardiovasc Interv 2013 [Epub ahead of print]; doi: 10.1002/ ccd.24984. 